\title{
Di che cosa si fa prevenzione? Delle malattie o della salute? Del male o del bene?
}

Vittorio Coletti

PUBBLICATO: 8 GIUGNO 2021

\section{Quesito:}

Alcuni lettori chiedono chiarimenti sul termine prevenzione e su quale siano i contesti più opportuni in cui usarlo.

\section{Di che cosa si fa prevenzione? Delle malattie o della salute? Del male o del bene?}

I nostri lettori notano spesso usi impropri della lingua anche in documenti pubblici. Quello che segnalano di prevenzione di/della è particolarmente interessante. Cominciamo col dire che prevenzione (dal latino tardo praeventionem), come il verbo prevenire suo stretto parente, contiene nel proprio etimo la sostanza del suo significato: ciò che viene prima, l'atto di anticipare, di precedere qualcuno o qualcosa. Prevenzione ha oggi sostanzialmente due significati: uno, calcato sul francese prévention, che comporta soprattutto l'uso della parola al plurale, di pregiudizio, preconcetto, con valenza quindi negativa ("un giudizio senza, con troppe prevenzioni", "è uno pieno di prevenzioni", "non bisogna avere prevenzioni"); l'altro, etimologico, di atto di protezione, tutela di qualcosa prima che possa danneggiarsi o essere danneggiata: "è necessaria una capillare prevenzione", "non è stata fatta sufficiente prevenzione". L'Istituto Superiore della Sanità (Centro nazionale malattie rare) definisce "la prevenzione... un insieme di attività, azioni ed interventi attuati con il fine prioritario di promuovere e conservare lo stato di salute ed evitare l'insorgenza di malattie". Ai primi dell'Ottocento, Gian Domenico Romagnosi (citato dal GDLI) aveva scritto che "(la prevenzione) ha sol per oggetto di impedire l'avvenimento di un male o danno temuto, si dal canto delle cose che dal canto degli uomini”: definizione perfetta ancora oggi.

In entrambi i significati connessi alla parola c'è dunque un tratto negativo, presunto o temuto. In passato erano attestati (sempre dal GDLI) anche dei significati positivi, di previdenza, previsione, prefigurazione e persino di disposizione favorevole:

B. Davanzati (ante I6oo): "Questa sollecitata prevenzione arà cagionato tre beni: rallegrato il mondo, insegnato a me e guardato il libraio di danno". Bersezio (1876): "Sorrise fra sé e senti inumidirsi gli occhi in prevenzione della dolcezza di quegli amplessi".

ma oggi non circolano più. Il dato negativo è prevalente e sostanzialmente il solo. Del resto, è quello più vicino all'etimo (si cerca di prevenire più un danno che un vantaggio) e si trova tra quelli originari della parola in italiano nel XVII secolo (fonte GDLI):

Giovanni Grimani, Relazioni di ambasciatori veneti al senato: "A necessaria prevenzione de' mali incontri feci opportunamente il mio solito ricorso al Conte di Traumesdorf".

La valenza negativa resta forse un po' nascosta nell'uso assoluto della parola, in espressioni frequenti come "buona, lodevole prevenzione", in cui è valutata positivamente l'azione in sé di prevenire, premunirsi, ma proprio perché volta contro o a tutela da eventuali aspetti o fenomeni negativi. Più 
evidente è il tratto negativo che giustifica e richiede la prevenzione quando di essa si specifica l'oggetto: è di questo che ci chiedono conto i lettori. Infatti, se la prevenzione è specificata da un complemento, questo non può che indicare cose, situazioni negative o pericolose o dannose. Si fa prevenzione delle malattie, dei tumori, degli infortuni, degli incidenti, dei contagi, delle frane, dei reati, della violenza ecc. Non si può fare prevenzione di un bene, ma di un male, per evitarlo prima che arrivi. Quindi, per rispondere ai lettori, non si puó fare prevenzione della salute, perché la si fa delle malattie; non si fa prevenzione della trasparenza degli atti pubblici, perché la si fa della loro opacità. Non si fa prevenzione dell'onestà, ma dei crimini; tant'è vero che negli "istituti di prevenzione (e pena)" (carceri) non ci sono gli innocenti ma i (presunti) colpevoli.

Domanda. Come mai cominciano a circolare complementi "positivi" di prevenzione come quelli (salute, trasparenza) segnalati dai lettori? Intanto, come segnala uno di loro, c’è uno slittamento semantico propiziato da una sinonimia approssimativa: siccome la prevenzione protegge $d a$ un danno, viene accostata, come sinonimo, a difesa, protezione: parole che, però, diversamente da essa, ammettono complementi sia negativi, con la preposizione da ("difesa, protezione dal raffreddore"), che positivi, condi ("difesa, protezione della salute"), cosa che non si dà per prevenzione (è ammessa solo "la prevenzione del raffreddore"). Quindi una sinonimia imperfetta, imprecisa, da evitare, fonte di equivoco nell'uso della nostra parola.

Poi, c'è un'altra ragione: lo scambio dell'effetto per la causa. Siccome (causa) la prevenzione previene i malanni e quindi protegge la salute (effetto), le si attribuisce la proprietà dell'effetto che invece non possiede. È un caso di rovesciamento di prospettiva. Sul nostro sito abbiamo qualche tempo fa segnalato un fenomeno analogo, quando abbiamo fatto notare che sui pannelli delle autostrade a volte si legge "controllo mancato pagamento pedaggio" e abbiamo ricordato che non si controlla una mancanza, ma un adempimento (per sanzionare l'eventuale omissione). Il controllore non chiede chi non ha il biglietto, ma controlla che tutti lo abbiano. In quel caso si introduce un complemento (di controllo) semanticamente non compatibile, specificandolo con un'assenza invece che con una presenza. Nel caso di prevenzione si fa una cosa simile: la si specifica con un complemento positivo, invece che negativo. Lo scostamento semantico è vistoso e scorretto, culturalmente più che grammaticalmente inesatto. Ma il rischio che si affermi è forte, come mostra il caso dei pannelli autostradali che nessuno ha mai pensato di correggere.

Casi come questi, che non riguardano la forma, ma la semantica, sono più insidiosi di quelli puramente formali: "prevenzione della salute" è grammaticalmente corretto, come "prevenzione dei tumori", ma la seconda espressione è ammissibile e la prima no, perché rischia di far passare la salute per qualcosa di dannoso per evitare il quale è bene fare prevenzione. Come "bere una bistecca" è grammaticalmente accettabile quanto "bere una birra", ma culturalmente non ammissibile, così "prevenzione di un bene" è inaccettabile, non per la grammatica, ma per la cultura.

\section{Cita come:}

Vittorio Coletti, Di che cosa si fa prevenzione? Delle malattie o della salute? Del male o del bene?, "Italiano digitale", XVII, 2021/2 (aprile-giugno)

DOI: $10.35948 / 2532-9006 / 2021.8555$

\section{Copyright 2021 Accademia della Crusca}

Pubblicato con licenza creative commons CC BY-NC-ND 